\title{
Atomic physics and quantum optics using superconducting circuits
}

\author{
J. Q. You ${ }^{1,2}$ and Franco Nori ${ }^{2,3}$ \\ ${ }^{1}$ Department of Physics, State Key Laboratory of Surface Physics, \\ and Key Laboratory of Micro and Nano Photonic Structures \\ (Ministry of Education), Fudan University, Shanghai 200433, China \\ ${ }^{2}$ Advanced Science Institute, RIKEN, Wako-shi 351-0198, Japan \\ ${ }^{3}$ Physics Department, The University of Michigan, Ann Arbor, MI 48109-1040, USA
}

\begin{abstract}
Superconducting circuits based on Josephson junctions exhibit macroscopic quantum coherence and can behave like artificial atoms. Recent technological advances have made it possible to implement atomic-physics and quantum-optics experiments on a chip using these artificial atoms. This review presents a brief overview of the progress achieved so far in this rapidly advancing field. We not only discuss phenomena analogous to those in atomic physics and quantum optics with natural atoms, but also highlight those not occurring in natural atoms. In addition, we summarize several prospective directions in this emerging interdisciplinary field.
\end{abstract}

Superconducting circuits with Josephson junctions can behave as artificial atoms. In these quantum circuits, the Josephson junctions act as nonlinear circuit elements (see Box 1). Such nonlinearity in a circuit ensures an unequal spacing between energy levels, so that the lowest levels can be individually addressed by using external fields (see, e.g., [1-9]). Experimentally, these circuits are fabricated on a micrometer scale and operated at $\mathrm{mK}$ temperatures. Because of the reduced dimensionality and thanks to the superconductivity, the environmentinduced dissipation and noise are greatly suppressed, so the circuits can behave quantum mechanically.

Superconducting circuits based on Josephson junctions have recently become subjects of intense research because they can be used as qubits - controllable quantum two-level system - for quantum computing (see, e.g., [14] for reviews). Even though the typical decoherence times of these circuits fall short of the requirements for quantum computation, their macroscopic quantum coherence is sufficient for them to exhibit striking quantum behaviors. These circuits can have a number of superconducting eigenstates with discrete eigenvalues lower than the energy levels of the quasi-particle excitations that involve breaking Cooper pairs. This property allows these circuits to behave like superconducting artificial atoms. Indeed, there is a deep analogy between natural atoms and the artificial atoms made from superconducting circuits (see Box 2). Both have discrete energy levels and can exhibit coherent quantum oscillations between these levels. Whereas natural atoms may be controlled using visible or microwave photons that excite electrons from one state to another, the artificial atoms in the circuits are driven by currents, voltages and microwave photons that excite the system from one macroscopic quantum state to another.

Differences between superconducting circuits and natural atoms include the different energy scales in the two systems, and how strongly each system couples to its environment; the coupling is weak for atoms and strong for circuits. In contrast to naturally occurring atoms, artificial atoms can be designed with specific characteristics and fabricated on a chip using standard lithographical technologies. With a view to applications, this degree of tunability is an important advantage over natural atoms. Thus, in a controllable manner, superconducting circuits can be used to test fundamental quantum mechanical principles at a macroscopic scale, as well as to demonstrate atomic physics and quantum optics on a chip. Moreover, artificial atoms can be designed to have exotic properties that do not occur in natural atoms.

\section{Box 1. The Josephson junction as a non- linear inductor}

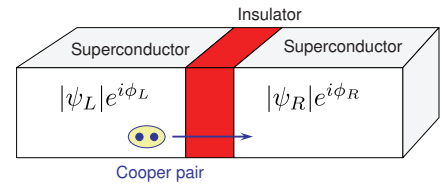

A superconductor contains many paired electrons, called Cooper pairs, which condense into the same macroscopic quantum state described by the wavefunction $|\psi| e^{i \phi}$, with $|\psi|^{2}$ being the density of Cooper pairs. In the absence of applied currents or magnetic fields, the phase $\phi$ is the same for all Cooper pairs. A Josephson junction is composed of two bulk superconductors separated by a thin insulating layer through which Cooper pairs can tunnel (see the figure above). The supercurrent through the junction is $I=I_{c} \sin \varphi$, where the critical current $I_{c}$ is related to the Josephson coupling energy $E_{J}$ of the junction by $I_{c}=(2 e / \hbar) E_{J}$, and $\varphi=\phi_{L}-\phi_{R}$ is the phase difference of the two superconductors across the junction. The time variation of this phase difference is related to the potential difference $V$ between the two superconductors: $\dot{\varphi}=\left(\frac{2 \pi}{\Phi_{0}}\right) V$, where $\Phi_{0}=h / 2 e$ is the magnetic-flux quantum. From the definition of the inductance $V=L_{J} \dot{I}$, it follows that $L_{J}=\frac{\Phi_{0}}{2 \pi I_{c} \cos \varphi}$, indicating that the Josephson junction behaves like a nonlinear inductor. 


\section{Box 2. Artificial and natural atoms}

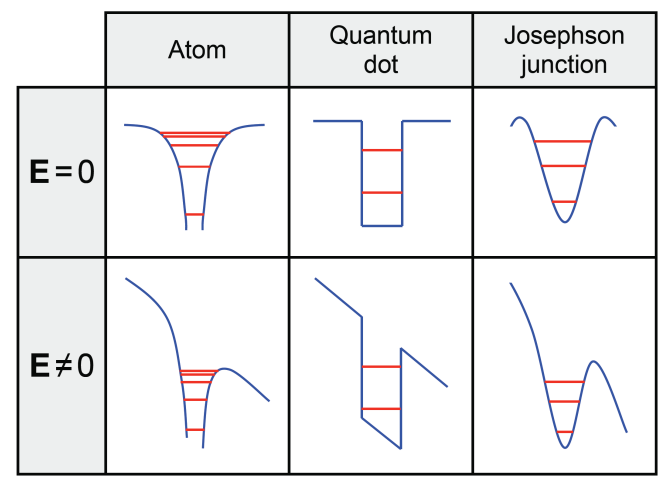

Potential energy (in blue) and discrete energy levels (in red) for an atom, a quantum dot and a Josephson junction in the absence $(\mathbf{E}=0)$ and presence $(\mathbf{E} \neq 0)$ of an externally applied electric field, respectively (see the figure above). Owing to their confinement, the electrons in the atom and the quantum dot have discrete energy levels. The Cooper pairs confined in the potential well of the Josephson coupling energy also have discrete energy levels and the junction can be regarded as a superconducting artificial atom.

In this review, we highlight the atomic-physics and quantum-optics phenomena found in superconducting circuits. The novel physics in these artificial atoms will be emphasized, including phenomena that do not occur in natural atoms. We also summarize several prospective directions in this emerging interdisciplinary field. Some of the examples in this brief overview relate to our work, because we are more familiar with them.

\section{Superconducting circuits as artificial atoms}

Two important energy scales determine the quantum mechanical behavior of a Josephson-junction circuit: The Josephson coupling energy $E_{J}$ and the electrostatic Coulomb energy $E_{c}=(2 e)^{2} / 2 C$ for a single Cooper pair, where $e$ is the electronic charge, and $C$ is either the capacitance $C_{J}$ of a Josephson junction or the capacitance of a superconducting island called a Cooper-pair box (i.e., the sum of the gate capacitance $C_{g}$ and the relevant junction capacitance), depending on the circuit. Figure 1 summarizes three kinds of superconducting circuits implemented in different regimes of $E_{J} / E_{c}$; Fig. 1(a) shows the voltage-driven box (also known as a Cooper-pair box) for a charge qubit [5], Fig. 1(b) the flux-driven three-junction loop for a flux qubit [6] and Fig. 1(c) the current-driven junction for a phase qubit $[8,9]$. As a typical example, energy levels of the flux qubit are shown in Fig. 1(d). Moreover, hybrid superconducting qubits are possible. For instance, a Cooper-pair box can behave like a chargeflux qubit [7] when $E_{J} / E_{c} \sim 1$. As for the flux qubit, by reducing the ratio $E_{J} / E_{c}$, the charge noise can become dominant over the flux noise [10] and the circuit behaves more like a charge qubit. In this circuit, when $\alpha<0.5$ (here $\alpha$ is the ratio of the Josephson coupling energy between the smaller and larger junctions in the loop), the double-well potential converts to a single-well potential and the circuit behaves like a phase qubit $[10,11]$. One can shunt a large capacitance to the small junction $[10,11]$ to suppress the charge noise in this circuit. Also, this large capacitance shunted to the Josephson junction can be used to reduce the charge noise in the Cooper-pair box [12], so as to implement the circuit in the phase regime. Below we highlight several aspects of the atomic-physics and quantum-optics phenomena found in superconducting circuits.

Cavity quantum electrodynamics. A quantized electromagnetic field can coherently exchange energy with a two-level system, usually in a tiny (micrometerscale) cavity. This energy exchange process involves a fundamental phenomenon called Rabi oscillation; the two-level system and the field exchange a quantum of energy back and forth at a characteristic frequency known as the Rabi frequency. When the field is in resonance with the system, the Rabi frequency is proportional to the system-field coupling strength. The most elementary of such coherent processes involves the interaction of a two-level system with a single photon in the cavity. The exchange of energy between the system and the single photon is observable when the Rabi frequency is larger than the decay rates of the two-level system and the cavity. This photon-atom coupling has been achieved for a variety of atoms interacting with the light field in a cavity and forms the basis of cavity quantum electrodynamics (QED). Cavity QED with superconducting circuits was proposed $[13,14]$ and experimentally achieved $[15,16]$ in systems where superconducting qubits were employed as two-level artificial atoms. For the cavity, a single-mode $L C$ resonator [15] and a multi-mode coplanar waveguide resonator [16] have been used. Significantly, the strongcoupling limit for a superconducting qubit in a cavity can be attained much more easily than for a natural atom in a cavity $[4,14]$ by suitably designing the system parameters. It allows, e.g., the observation of the Lamb shift for a superconducting qubit in the cavity [17]. Moreover, both the superconducting qubit and the cavity can be fabricated on the same chip. For a review on cavity QED with superconducting qubits, see, e.g., [4].

Because they can be designed with specific system parameters, superconducting quantum circuits are suited for achieving the so-called ultrastrong-coupling regime, where the qubit-photon coupling strength is comparable 


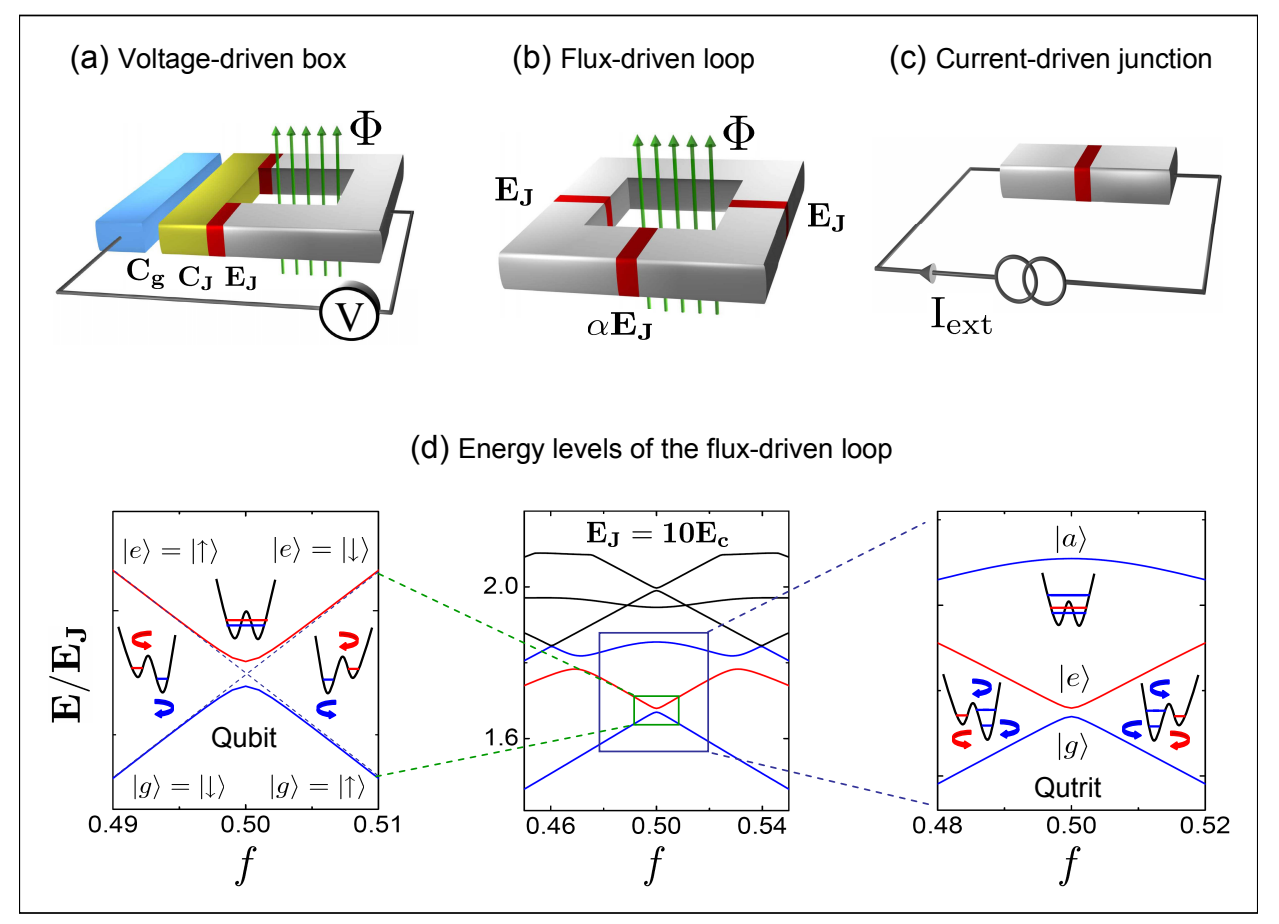

FIG. 1: Superconducting circuits as artificial atoms. (a) A Cooper-pair box biased by a gate voltage $V_{g}$ and implemented in the charge regime $E_{J} / E_{c} \ll 1$. The SQUID loop provides an effective Josephson coupling energy tuned by the threading magnetic flux $\Phi$. See main text for nomenclature. (b) A superconducting loop interrupted by three Josephson junctions and implemented in the phase regime $E_{J} / E_{c} \gg 1$. The two identical Josephson junctions have coupling energy $E_{J}$ and capacitance $C$, while both the Josephson coupling energy and the capacitance of the smaller junction are reduced by a factor $\alpha$, where $0.5<\alpha<1$. The three-junction loop is biased by a flux $\Phi$ such that $f \equiv \Phi / \Phi_{0} \sim \frac{1}{2}$. (c) A Josephson junction biased by a current $I_{\text {ext }}$, which is also implemented in the phase regime and has a much larger ratio $E_{J} / E_{c}$. (d) Energy levels of the flux-driven three-junction loop (the curves in the middle panel). With the lowest two energy levels involved (the curves in the left panel, which are enlarged from the smaller rectangle in the middle panel), the flux-driven loop can behave like a coherent and controllable quantum two-level system (qubit), while the circuit can behave like a coherent and controllable three-level system (qutrit) when using the lowest three levels (the curves in the right panel, which are enlarged from the larger rectangle in the middle panel). Moreover, in the left, top and right insets of the left (right) panel, the two (three) energy levels are also displayed in the double potential well for $f<\frac{1}{2}, f=\frac{1}{2}$ and $f>\frac{1}{2}$, where the clockwise and anticlockwise arrows represent the circulating supercurrent states in the flux-driven three-junction loop. In (a)-(c), the qubit employs the charge states on a single island, the persistent-current states in a double potential well, and the anharmonic-oscillator states in a single potential well, respectively. Also, a flux-driven superconducting loop with a different number of Josephson junctions, e.g., one [89] or four junctions [90], can be used for a flux qubit. Furthermore, a flux-driven single-junction loop can be used as a phase qubit when working with the energy levels in a tilted potential well [91], as in a current-driven junction.

to the energy scales of the qubit and the photon [18]. Indeed, there have been a number of theoretical studies of this system, analyzing some of its rich static and dynamical properties (see, e.g., [19-21]). Also, the ultrastrong coupling between a superconducting flux qubit and a coplanar waveguide [22] or an $L C$ resonator [23] has recently been demonstrated in experiments. One can expect to find new phenomena in this ultrastrong-coupling regime which are not present in the conventional weakand moderately strong-coupling regimes. In addition, dressed states of a superconducting charge qubit and an intense microwave field were experimentally observed by embedding the circuit in an $L C$ oscillator [24]. The tunability of these dressed states allows one to explore both resonant and dispersive coupling regimes.
Selecting quantum transitions. In natural atoms, the electronic state at each orbital level has a well-defined parity symmetry, either even or odd. Under the dipole approximation, the interaction Hamiltonian between the atom and the time-dependent electric field has an odd parity. Thus, to have a nonzero dipole transition matrix element, there should be a parity change between the initial and final states, in addition to the constraints on azimuthal and magnetic quantum numbers of the electronic states. This optical selection rule dictates that only three types of three-level systems, called $\Lambda$-, $V$ , and $\Xi$-type atoms [see Fig. 2(a)], exist for the natural atoms, where no dipole transition between electronic states with the same parity are allowed. However, selection rules can be different for superconducting artificial 


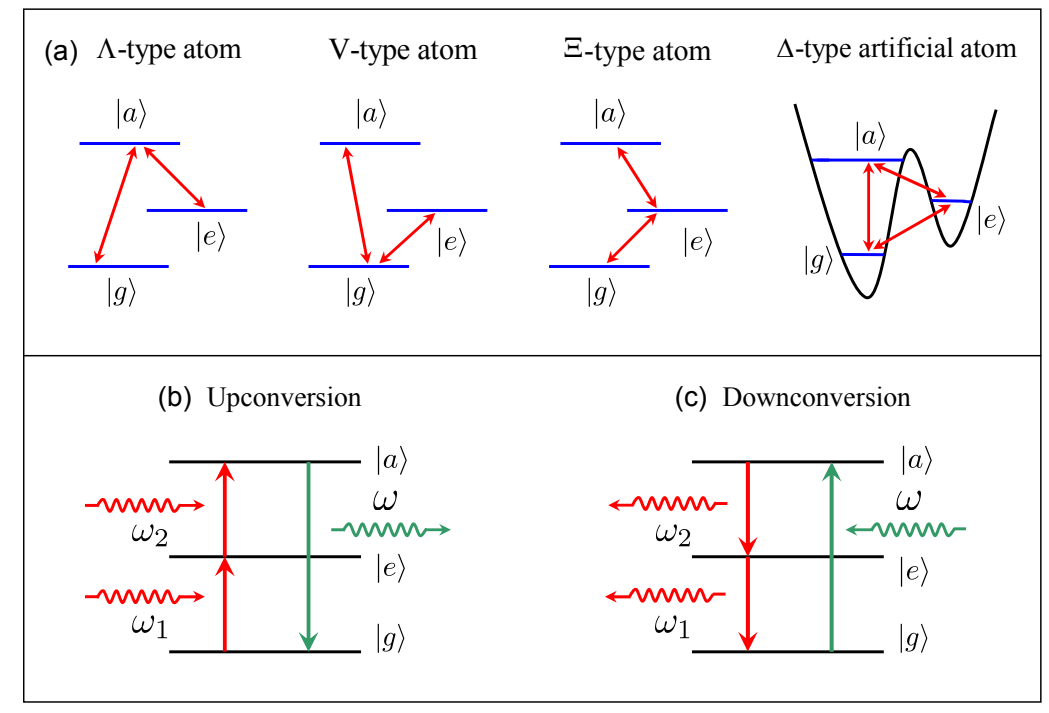

FIG. 2: Three-level atoms and frequency conversion. (a) Energy levels of natural atoms of the $\Lambda, V$, and $\Xi$ types, as well as a $\Delta$-type artificial atom consisting of a flux-driven three-junction loop. The allowed dipole transitions between energy levels are indicated in red. In contrast to naturally occurring atoms, the three dipole transitions among the states $|g\rangle$, $|e\rangle$ and $|a\rangle$ are all allowed in the $\Delta$-type three-level artificial atom. Here $|g\rangle$ and $|e\rangle$ denote the ground and first excited states, while $|a\rangle$ denotes either the second or another excited state. (b) Frequency upconversion in a $\Delta$-type artificial atom. Here $\omega_{1}=\left(E_{e}-E_{g}\right) / \hbar, \omega_{2}=\left(E_{a}-E_{e}\right) / \hbar$, and $\omega=\left(E_{a}-E_{g}\right) / \hbar$, with $E_{i}(i=g, e$ or $a)$ being the energy level of the state $|i\rangle$. When two microwave photons, one with frequency $\omega_{1}$ and the other with $\omega_{2}$, are successively absorbed by the artificial atom, it can emit a microwave photon with frequency $\omega=\omega_{1}+\omega_{2}$ via the transition $|a\rangle \rightarrow|g\rangle$. (c) Frequency downconversion in the $\Delta$-type artificial atom. When a microwave photon with frequency $\omega$ is absorbed by the artificial atom, the sequential transitions $|a\rangle \rightarrow|e\rangle$ and $|e\rangle \rightarrow|g\rangle$ can produce two microwave photons with frequencies $\omega_{1}$ and $\omega_{2}$, respectively. In particular, when $E_{a}-E_{e}=E_{e}-E_{g}$, the upconversion in (b) converts two photons with frequency $\frac{1}{2} \omega$ to one photon with frequeny $\omega$, while the downconversion in (c) converts one photon with frequency $\omega$ to two photons with frequeny $\frac{1}{2} \omega$. Natural atoms cannot perform up- or down-conversion, unless aided by nonlinear effects. However, artificial atoms can.

atoms. For instance, in the dipole approximation, the interaction Hamiltonian between a flux qubit circuit and a time-dependent magnetic field does not have a welldefined parity, except at the point with a static magnetic flux $f \equiv \Phi / \Phi_{0}=\frac{1}{2}$, where $\Phi$ is the static magnetic flux applied to the loop and $\Phi_{0}$ the magnetic-flux quantum. At this particular point, the interaction Hamiltonian has an odd parity. Owing to the parity symmetries of the artificial-atom states at $f=\frac{1}{2}$, the lowest three levels of the circuit behave like a $\Xi$-type or laddertype artificial atom [25]. In this case, the dipole transition between $|g\rangle$ and $|a\rangle$ is forbidden, while the other two transitions (among states $|g\rangle,|e\rangle$ and $|a\rangle$ ) are allowed [see Fig. 2(a) for nomenclature]. However, when $f \neq \frac{1}{2}$, the parity symmetry is broken for the interaction Hamiltonian. Therefore, all three dipole transitions among $|g\rangle,|e\rangle$ and $|a\rangle$ are possible, allowing the atom to be $\Delta$-type because of the triangle-shaped transitions among the three energy levels. Now (when $f \neq \frac{1}{2}$ ) the superconducting circuit behaves as a $\Delta$-type cyclic artificial atom, where one- and two-photon processes can coexist [25].

This $\Delta$-type artificial atom can be used for the upconversion and downconversion of the photon frequency [see
Figs. 2(b) and 2(c)]. In these frequency conversions, all transitions involve only linear processes; this is in sharp contrast to the conventional frequency conversion in nonlinear optics, where a nonlinear medium is used and the nonlinear effect facilitates converting the frequency of the photons. Recently, the frequency upconversion of a microwave photon was experimentally demonstrated in a flux qubit [26]. This experiment explained the observed coexistence of one- and two-photon processes as due to the symmetry-breaking of the system Hamiltonian, when varying the applied magnetic flux away from $f=\frac{1}{2}$.

In the experiment reported in [27], two microwave fields were applied simultaneously to a superconducting circuit containing two coupled flux qubits. The interference between the processes that correspond to a selected excitation by the applied microwave fields can be controlled and used to activate or suppress a given transition. Thus, this method effectively creates artificial and controllable selection rules.

Electromagnetically induced transparency. Quantum interference can be introduced to control the propagation of light through an atomic medium consisting of three-level atoms or qutrits. We now consider a $\Lambda$-type three-level atom; to control the propagation of a 

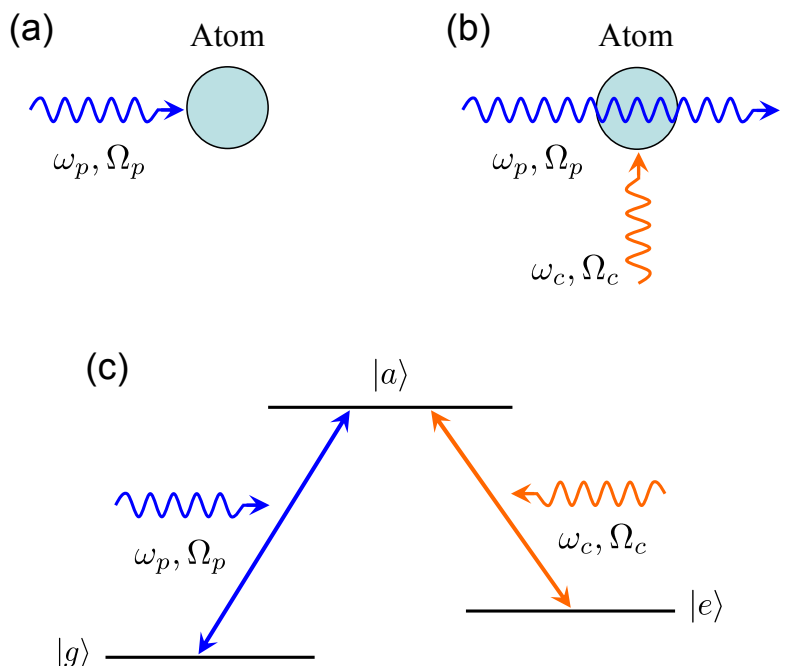

FIG. 3: Electromagnetically induced transparency. (a) A probe light field is absorbed by natural or artificial atoms when the frequency of the light field is resonant with a particular separation between two atomic energy levels. (b) However, the probe light field can go through the natural or artificial atoms when a suitable control light field also drives the atoms. (c) $\Lambda$-type three-level atomic system for EIT. The frequency $\omega_{p}$ of the probe light field is resonant with the energy separation between states $|a\rangle$ and $|g\rangle$, and the frequency $\omega_{c}$ of the control light field is resonant with the energy separation between states $|a\rangle$ and $|e\rangle$. The Rabi frequency $\Omega_{p}\left(\Omega_{c}\right)$ quantifies the coupling strength between the probe (control) light field and the atom.

probe light field in resonance with the dipole transition $|a\rangle \leftrightarrow|g\rangle$ (that is, at the probe frequency $\omega_{p}$ ), one can drive the atom by a second (control) field that is in resonance with the transition $|a\rangle \leftrightarrow|e\rangle$ at the control frequency $\omega_{c}$ (see Fig. 3). Now the amplitude of the wavefunction for the state $|a\rangle$ is driven by two terms; one proportional to the probability amplitude of the ground state $|g\rangle$ and the other proportional to the probability amplitude of state $|e\rangle$. The combined effect of these two fields is to pump the atom into a coherent superposition of the states $|g\rangle$ and $|e\rangle$ known as a dark state. In such a case, the two driving terms can interfere and cancel each other, i.e., they have equal magnitudes but opposite signs. Under this destructive quantum interference, the occupation probability at state $|a\rangle$ is zero, leading to a vanishing light absorption even in the presence of light fields. This effect is called electromagnetically induced transparency (EIT) in quantum optics [28, 29] and also applies to $V$ - and $\Xi$-type atoms. This important effect has a variety of applications. For instance, a medium with EIT can slow down and even stop or trap light.

We now consider EIT in a more quantitative manner. Let $\Omega_{p}\left(\Omega_{c}\right)$ be the Rabi frequency that quantifies the coupling strength between the probe (control) light and the atom. Here we assume that a $\Lambda$-type atom can be prepared in the initial state $|\Psi(0)\rangle=\left(\Omega_{c}|g\rangle-\Omega_{p}|e\rangle\right) / \Omega$, where $\Omega=\sqrt{\Omega_{p}^{2}+\Omega_{c}^{2}}$. When the EIT occurs, the atom will be trapped in this dark state, i.e., $|\Psi(t)\rangle=$ $\left(\Omega_{c}|g\rangle-\Omega_{p}|e\rangle\right) / \Omega$, for a time which is dependent on the decoherence rate of the atom. Usually, it is not easy for an atom to be prepared in the initial state $|\Psi(0)\rangle$, when $\Omega_{p}$ is comparable to $\Omega_{c}$. Instead, the atom can be naturally prepared in the ground state $|g\rangle$. If strong control and weak probe fields are chosen so that $\Omega_{c} \gg \Omega_{p}$, the dark state $|\Psi(t)\rangle$ is close to the initial state $|g\rangle$. In such a case, the combined action of the control and probe fields can easily drive the atom from the ground state into the dark state [29]. This is the reason why a strong control field and a weak probe field are used to experimentally implement EIT in an atomic medium.

At $f=\frac{1}{2}$, the flux qubit circuit can behave like a $\Xi$ type artificial atom $[25,30]$. The circuit can also behave approximately like a $\Lambda$-type artificial atom when $f \neq \frac{1}{2}$, if the dipole transition rate between $|g\rangle$ and $|e\rangle$ is much smaller than the rates for the other two transitions [30]. As discussed above, these selection rules are related to the parity symmetries of the artificial-atom states. In contrast to natural atoms, the tunability of a superconducting circuit can be used to prepare the artificial atom in an initial state that is close to the dark state $|\Psi\rangle$ with arbitrary $\Omega_{c}$ and $\Omega_{p}$. Therefore, it is experimentally feasible to produce EIT in a single artificial atom for either strong or weak control and probe fields. This is an important advantage of superconducting circuits compared to natural atoms. EIT using superconducting circuits has been studied theoretically (e.g., [31-33]) and experimentally $[34,35]$. In [35], this phenomenon was experimentally shown using a four-junction loop biased at $f=\frac{1}{2}$, where the circuit behaves as a $\Xi$-type artificial atom.

State population inversion and lasing. A laser is composed of an amplifying medium inside a resonant optical cavity. When the system is driven, a state population inversion (SPI) can be achieved for the atoms or molecules in the amplifying medium. Moreover, there is a positive feedback between the emitted light and the amplifying medium. Because of this positive feedback and the nature of the stimulated photon emission, the laser has a large net optical gain and the emitted photons have the same direction, phase and polarization. These advantages mean that the laser has a variety of applications in different fields. Recently, several studies have considered lasing using only a single artificial atom, both theoretically [30, 36-38] and experimentally [39, 40].

With suitable junction parameters, the flux qubit circuit can have the following dipole transition rates [30] when $f \neq \frac{1}{2}: \Gamma_{g a}>\Gamma_{a e} \gg \Gamma_{e g}$, where the rate $\Gamma_{i j}$ is proportional to $\left|t_{i j}\right|^{2}$, with $t_{i j}$ being the dipole transition matrix element between states $|i\rangle$ and $|j\rangle$. Because the transition $|a\rangle \rightarrow|e\rangle$ can be dominant over $|e\rangle \rightarrow|g\rangle$, an 
(a)
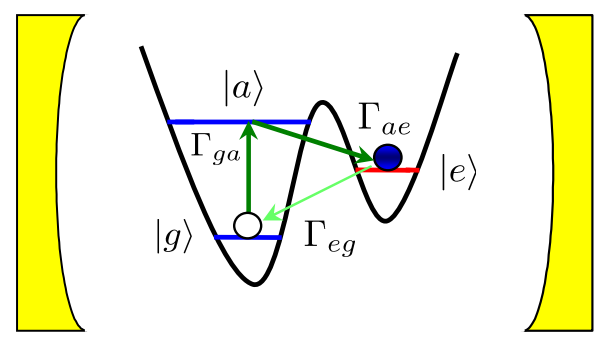

(b)
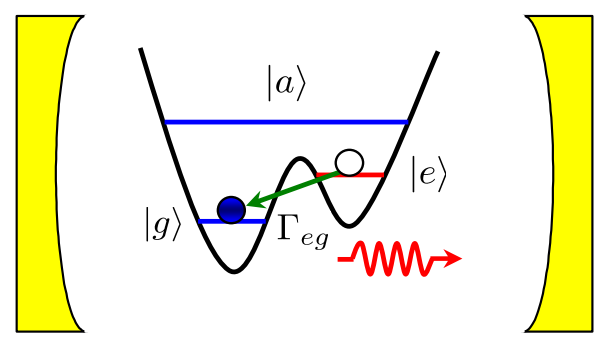

FIG. 4: Lasing. (a) State population inversion (for lasing) between states $|e\rangle$ and $|g\rangle$ in a three-junction loop at $f>$ $\frac{1}{2}$, where the artificial atom is quickly pumped from $|g\rangle$ to $|a\rangle$ by a strong microwave pulse (for example, by a quick Rabi oscillation) and then decays from $|a\rangle$ to $|e\rangle$ via photon emission. Here the dipole transition rate from $|e\rangle$ to $|g\rangle$ is small because of a higher inter-well barrier between them; the dipole transition rate from $|a\rangle$ to $|e\rangle$ is larger owing to a lower barrier; and the rate is even larger for the dipole transition $|g\rangle \rightarrow|a\rangle$ because no potential barrier is involved in the single well. (b) Photon emission of the artificial atom to build up a lasing field, where the inter-well barrier is lowered so as to have a strong transition rate between $|e\rangle$ and $|g\rangle$ and to tune the transition $|e\rangle \rightarrow|g\rangle$ into resonance with the cavity.

SPI between states $|e\rangle$ and $|g\rangle$ [Fig. 4(a)] can be quickly achieved by pumping the artificial atom (via the transition $|g\rangle \rightarrow|a\rangle)$ using a strong microwave field. Here the artificial atom can be placed in, e.g., a coplanar waveguide resonator [16] by sharing a segment of the circuit loop with the transmission line. In the process of SPI, the weak-coupling regime is assured by having the flux qubit circuit off-resonant with respect to the cavity.

While the SPI is being established, the biasing flux can be adjusted to give a value of $f$ near $\frac{1}{2}$, where the rate $\Gamma_{e g}$ for the transition $|e\rangle \rightarrow|g\rangle$ becomes large and the cavity is resonant with this transition. However, the adiabatic condition is not easy to satisfy near this anticrossing point $\left(f=\frac{1}{2}\right)$, where the Landau-Zener transition is strong, so the biasing flux cannot be changed very fast while approching this point. Fortunately, away from this anticrossing point, the Landau-Zener transition is weak, so it is easy to satisfy the adiabatic condition [30] and the flux can be changed very quickly. To take advantage of this property, the small Josephson junction in the fluxdriven loop can be replaced by a tunable superconducting quantum interference device (SQUID). In this d.c.
SQUID, the magnetic field applied to the loop causes the critical current to oscillate with period $2 \Phi_{0}$. With the SPI established at a biasing flux away from $f=\frac{1}{2}$, one can quickly change the flux in the SQUID loop to lower the inter-well barrier, so as to both increase the transition rate $\Gamma_{e g}$ and tune this transition $|e\rangle \rightarrow|g\rangle$ into resonance with the cavity mode [Fig. 4(b)]. This can yield a strong coupling between the circuit and the cavity.

To build up a lasing field, in addition to the above two conditions for quickly establishing SPI and then achieving a strong circuit-cavity coupling, the cavity used should have a high quality factor $Q$ ( $Q$-factor), i.e., a small leak or decay rate. This can be implemented using a coplanar waveguide resonator [16].

Indeed, lasing was experimentally observed using a Cooper-pair box in an on-chip cavity [39]. In contrast to the proposal [30] using a flux-driven loop, this experiment [39] employs a different three-level system: the two lowest superconducting states $|g\rangle$ and $|e\rangle$ and a quasiparticle state $|a\rangle$. The gate voltage is tuned to $V_{g}>e / C_{g}$ (above the degeneracy point), so the state $|2\rangle$ with an extra Cooper pair in the box becomes the ground state $|g\rangle$ of the artificial atom, and the state $|0\rangle$ with zero extra Cooper pair in the box is the excited state $|e\rangle$. Also, the box is connected to a lead via a tunnel barrier. When driving the box with a voltage across the tunnel barrier, an SPI between $|e\rangle \equiv|0\rangle$ and $|g\rangle \equiv|2\rangle$ is achieved, following quasi-particle tunneling processes [36]. In [39], lasing was achieved continuously, with emitted light escaped from one end of the cavity.

Cooling. There are different methods for cooling atoms, including Dopper cooling, Sisyphus cooling, sideband cooling, subrecoil cooling, and evaporative cooling. Some of these techniques can be adapted to cool a solidstate artificial atom. For instance, the Sisypus cooling technique was used to cool a flux qubit (i.e., a flux-driven three-junction or four-junction loop) [40].

Another important advancement [42] is the cooling of a flux qubit implemented via the inverse process of SPI. In [42], the temperature of the superconducting qubit was lowered by up to two orders of magnitude when its surroundings reached a temperature as low as tens of $\mathrm{mK}$. This cooling of the flux qubit is achieved when the biasing flux is shifted away from $f=\frac{1}{2}$, where the dipole transition rates for the lowest three levels of the flux qubit satisfy the relations $\Gamma_{a g}>\Gamma_{e a} \gg \Gamma_{g e}$. By optically pumping the qubit via the transition $|e\rangle \rightarrow|a\rangle$, the qubit is excited to the high-energy state $|a\rangle$ and then decays to the ground state by way of the transition $|a\rangle \rightarrow|g\rangle$ [see Fig. 5(a)], with a net energy, extracted from the qubit, emitted to the outside environment. This experiment also provides fine analogies between solid-state artificial atoms and natural atoms, as well as showing how these analogies can inspire new applications.

Although the superconducting qubit in [42] was greatly 

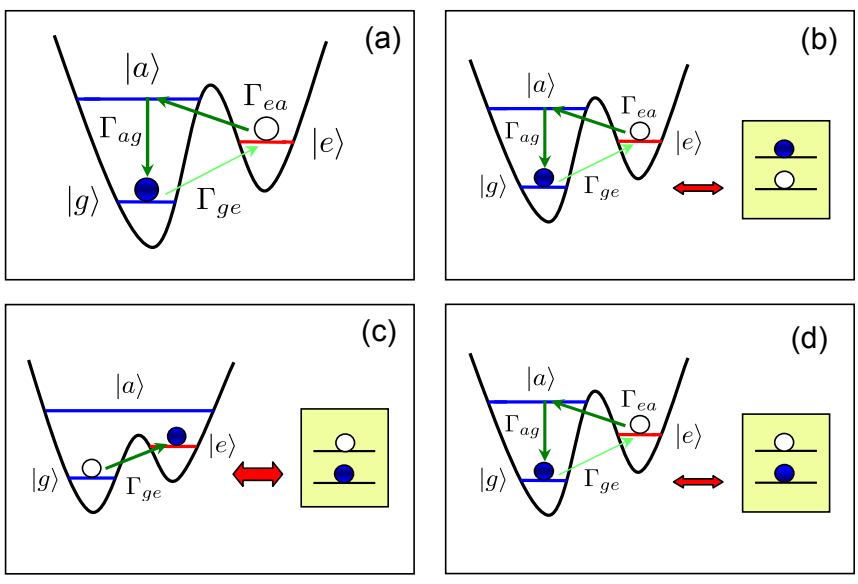

FIG. 5: Cooling a three-level artificial atom and a nearby two-level system. (a) Cooling the three-junction loop to its ground state $|g\rangle$. While the artificial atom is thermally excited to $|e\rangle$, one can drive the atom to $|a\rangle$ by a microwave field. Because of a large transition rate for $|a\rangle \rightarrow|g\rangle$, the atom can decay quickly from the unstable state $|a\rangle$ to the ground state, emitting net energy, extracted from the atom, to the outside environment. The levels in the double potential well correspond to the lowest three energy levels of a threejunction loop biased at $f>\frac{1}{2}$. (b) While the noise source is thermally excited, the artificial atom is shifted off-resonance to the noise source by tuning the externally applied flux and also driven to the cooled state via the inverse process of the state population inversion in (a). The box to the right of the energy level diagram represents a two-level fluctuator acting as a noise source. (c) The inter-well barrier of the artificial atom is lowered by tuning the externally applied flux so as to have both a strong transition rate between $|g\rangle$ and $|e\rangle$ and the transition $|g\rangle \rightarrow|e\rangle$ in resonance with the two-level system, so as to extract energy from the two-level system. (d) Shifting the artificial atom off-resonance from the two-level system and cooling the atom again, with the net energy extracted from the two-level system emitted to the outside environment.

cooled $\left(k_{B} T \ll E_{e}-E_{g}\right)$ in experiments, the noise sources surrounding the qubit were not. So the qubit will quickly return to the temperature of its environment. To overcome this difficulty, the superconducting qubit can be redesigned to increase its controllability by replacing the small Josephson junction in the flux-driven loop with a tunable SQUID [43]. The cooling process can now be described as follows [see Figs. 5(b)-5(d)]: First, as in the experiment [42], the qubit is initially cooled, following the inverse process of the SPI. Then, the tunable qubit is switched on for a period of time in order to resonantly interact with the noise source (e.g., local two-level fluctuators) surrounding the qubit. This process extracts energy from the noise source to heat the qubit. Repeating these two processes [43], both the qubit and its neighboring noise source can be simultaneously cooled. This will significantly enhance the quantum coherence of the qubit, because the cooled qubit is then thermally activated only very slowly to the first excited state.
Recent technical advances allow the fabrication of a nanomechanical resonator with both a high $Q$-factor and a sufficiently high frequency, close to the typical frequencies of superconducting circuits [44]. This has stimulated researchers to propose different ways to use superconducting circuits to achieve the ground-state cooling of coupled nanomechanical resonators [37, 43, 45-48]. Moreover, the experimental cooling of such a resonator, by coupling it to a superconductor single-electron transistor [49] or to a microwave-frequency superconducting resonator [50], has also been reported. When a nanomechanical resonator is cooled to the ground state [51], it provides a good platform for exploring various quantum phenomena and for observing the quantum-to-classical transition in such a macroscopic object. This will give rise to the new subject of quantum acoustics.

Photon generation. Superconducting qubits have the advantage of manipulating quantum states in a controllable manner. If these stationary qubits are spatially separated, one can use single photons generated in an extended cavity as a quantum bus, similar to a flying qubit, to implement quantum communication among them [see Fig. 6(a)]. Technologically, this requires the generation of single photons by manipulating a superconducting qubit and the transfer of information between the superconducting qubits and the photons. Using an on-chip cavity, it becomes feasible to achieve this quantum communication process on a chip.

Recent experiments show that a single-photon source can be achieved using a superconducting qubit coupled to an on-chip cavity [52-55]. When the qubit is prepared in the excited state $|e\rangle$ by a control pulse, it can decay to the ground state $|g\rangle$ by emitting one (and only one) photon in the on-chip cavity; this decay is possible because of the interaction between the qubit and the cavity. If the cavity was originally in the vacuum state $|0\rangle$, it now changes to the single-photon state $|1\rangle$. When the qubit is prepared in an arbitrary superposition state $\alpha|g\rangle+\beta|e\rangle$, in an ideal manner, the coupling between the qubit and the cavity can map the qubit state into a superposition state of zero and one photon in the cavity: $\alpha|0\rangle+\beta|1\rangle$ [see Figs. 6(b)-6(d)]. Furthermore, the experiment in [52] shows how to transfer the information from a cavity to a stationary qubit [see Figs. 6(d)-6(f)]. These experiments demonstrate that both single-photon sources and quantum communication between superconducting qubits can be achieved on a chip. However, because of the relaxation and decoherence in both qubit and cavity, after each step for transferring the information between a stationary qubit and a cavity, the amplitudes $\alpha$ and $\beta$ of the photon (qubit) state can be different from the amplitudes $\alpha$ and $\beta$ of the previous qubit (photon) state. Improving relaxation rates and qubit decoherence times would allow higher-fidelity state transfers between qubits and cavities. 


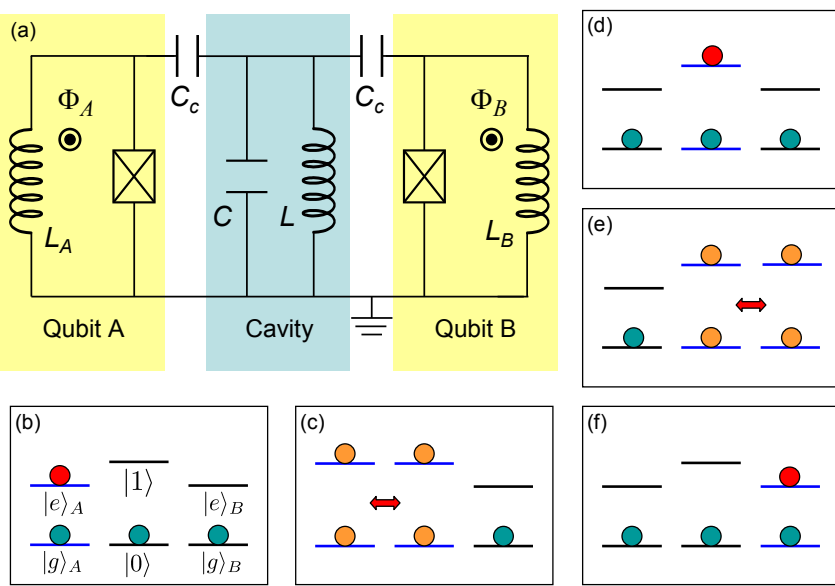

FIG. 6: Transferring quantum information between two stationary qubits via a cavity. (a) Schematic diagram of two flux-driven phase qubits capacitively coupled by an on-chip cavity (an $L C$ resonator). (b) Qubit A is prepared in a superposition state $\alpha|g\rangle_{A}+\beta|e\rangle_{A}$, while both qubit $\mathrm{B}$ and the resonator are prepared in their ground states. In this step, both qubits A and B are off-resonance with the cavity. (c) Qubit A is shifted into resonance with the resonator, for a time interval $t_{1}=\pi / 2 g_{A}$, with $\hbar g_{A}$ being the interaction energy between qubit $\mathrm{A}$ and the resonator. This step maps the state of qubit A to the superposition state $\alpha|0\rangle+\beta|1\rangle$ of the resonator, where $|0\rangle$ and $|1\rangle$ are two Fock states of the resonator with zero and one photon, respectively. (d) Shift qubit A off-resonance with the resonator again, and store the quantum information in the resonator for a time duration $t_{2}$. (e) Shift qubit $B$ in resonance with the resonator for a time interval $t_{3}=\pi / 2 g_{B}$, where $\hbar g_{B}$ is the interaction energy between qubit $B$ and the resonator. This step maps the state of the resonator to the superposition state $\alpha|g\rangle_{B}+\beta|e\rangle_{B}$ of qubit B. (f) Shift qubit B off-resonance with the resonator again, and store the quantum information in qubit B. Note that a high-fidelity state transfer between qubits A and B can be implemented if both the relaxation and decoherence of the state are negligibly small during the above processes.

In addition to single-photon generation, one can also generate, as proposed in [56, 57], multi-photon Fock states $|n\rangle$ (i.e., the number states of photons) and arbitrary superposition states $\sum_{n} c_{n}|n\rangle$. Indeed, in a recent experiment [58], the controlled generation of pure Fock states with up to 15 photons was achieved using a superconducting phase qubit coupled to a microwave on-chip cavity. Moreover, thanks to the advantages of both onchip cavity and tunable superconducting circuits, complex superpositions of states with different number of photons were also generated in a controlled and deterministic manner [55], which is a beautiful experimental realization of the protocol described in [56]. Recently, the $N$-photon entangled NOON states, $|N 0\rangle+|0 N\rangle$, have also been generated in two superconducting resonator [59]. These experiments further reveal the quantum behavior of the on-chip cavity and provide a useful on-demand multi-photon source for future quantum-technology ap- plications.

Quantum state tomography. A crucial step in quantum information processing is the measurement of the output quantum states. However, a quantum state cannot be ascertained by a single quantum measurement. This is because quantum states may comprise many complementary features which cannot be measured simultaneously and precisely, owing to uncertainty relations. Nevertheless, all complementary aspects can in principle be observed by a series of measurements on a large enough number of identically prepared copies of the quantum system. Then, one could reconstruct an unknown quantum state from such a complete set of measurements of system observables. Such a process of reconstructing quantum states is called quantum state tomography. Using state tomography, the noisy channel of the quantum system can also be determined. This procedure of determining the dynamics of an open quantum system is known as quantum process tomography.

Tomographic measurements on the quantum states of superconducting charge qubits, either single or multiple qubits, were proposed in [60]. Recently, there were many experiments on the quantum state tomography of single superconducting phase qubits $[61,62]$ and of two coupled superconducting phase [63] and charge [64] qubits. Also, quantum process tomography was experimentally implemented on single [65] and two [66] phase qubits. Indeed, quantum state tomography is an essential tool in qubitstate measurements, and quantum process tomography can be used to probe the noise properties and temporal dynamics of qubit systems.

\section{Future prospects}

With technological advances, superconducting circuits can be used to test quantum mechanics on a macroscopic scale (see Box 3). Also, they can be used to demonstrate many novel phenomena in quantum science. A few examples are listed below.

Dynamical Casimir effect. When two mirrors are placed in empty space, their presence affects the vacuum fluctuations of the electromagnetic field. Because of the different densities of the vacuum modes inside and outside of the space between the two mirrors, a net force on the mirrors can be generated. This effect of quantum electrodynamics is known as the static Casimir effect.

If the mirrors move, there is also a mismatch between vacuum modes at different times. It has been predicted that this may result in the creation of real photons out of vacuum fluctuations. This dynamical Casimir effect also holds for a single mirror subject to a nonuniform acceleration in empty space. Although receiving considerable interest since its theoretical prediction, there is still no 
experimental verification of the dynamical Casimir effect. This is mainly due to the fact that the rate of photon production is non-negligible only when the mirror velocity approaches the speed of light, making the use of massive mirrors very challenging. A coplanar waveguide terminated by a SQUID was proposed [67] for experimentally observing the dynamical Casimir effect. Changing the magnetic flux threading the SQUID loop parametrically modulates the boundary condition of the waveguide and thereby its effective length. Because there is no massive mirror moving, the velocity of the effective boundary can approach the speed of light. Photon production from the vacuum can thus be made experimentally detectable.

Coherent population transfer. Elementary logic gates in quantum computing networks are usually implemented using precisely-designed resonant pulses. However, the various fluctuations and operational imperfections that exist in practice limit these designs. Also, the difficulty of switching interqubit couplings on and off strongly limits the precise design of the required pulses for two-qubit gates. To overcome these difficulties, Ref. [68] proposes an approach to coherently transfer the populations of qubit states by using Stark-chirped rapid adiabatic passages. As in the case of geometric phases, these population transfers are insensitive to the dynamical evolution times of the qubits, as long as they are adiabatic. The rapid adiabatic passages of populations could offer an attractive approach to implementing high-fidelity single- and two-qubit gates for quantum computing.

The key of these rapid adiabatic passages is how to produce time-dependent detunings by chirping the qubit levels. For most natural atomic or molecular systems, where each bound state possesses a definite parity, the required detuning chirps could be achieved by making use of the Stark effect via, eg., two-photon excitations of the qubit levels [69]. The breaking of parity symmetries in the bound states in superconducting circuits such as current-biased Josephson junctions provides an advantage [68], because the desirable detuning chirps can be produced by single-photon pulses. Recently, rapid adiabatic passage was achieved for the tranfer of a single photon in a superconducting circuit [70].

Tunable mirrors and interferometers. Superconducting circuits can be used for Landau-ZenerStückelberg interferometry [71], but they can also be used for other types of interferometry -including Fano and Fabry-Perot interferometry [72, 73] — by coupling superconducting qubits to a coplanar waveguide. When injected into the waveguide, the photons interact with the qubits along the way, controlled by changing the applied electric and/or magnetic fields on the qubits. These artificial atoms, working as tunable mirrors, can change the reflection and transmission coefficients of the photons confined in the waveguide.

\section{Box 3. Testing quantum mechanics with macroscopic superconducting circuits}

\section{Bell inequality}

The Bell inequality shows that the predictions of quantum mechanics can contradict those of local hidden variable theories (see, e.g., [92]) if one looks at correlations between spatially separated measurements. It can alternatively be stated that no physical theory of local hidden variables can reproduce all of the predictions of quantum mechanics. Tests of the Bell inequality have been proposed, using superconducting circuits such as charge [93] and phase qubits [94]. Recently, the violation of the Bell inequality has been experimentally verified [95] in phase qubits. Because the Bell inequality is violated by a quantum mechanical prediction, this experiment provides strong evidence that these macroscopic superconducting circuits indeed behave quantum mechanically. Recent experimental results $[96,97]$ on Greenberger-Horne-Zeilinger states do not require statistical arguments for a violation of the Bell inequality to be seen.

\section{Leggett-Garg inequality}

Leggett and Garg derived an inequality for a single degree of freedom undergoing coherent oscillations and being measured at successive times [98]. The LeggettGarg inequality can be regarded as a temporal version of Bell's inequality, and it should be violated by a quantum two-level system. Very recently, this has been verified experimentally [99] using a voltage-driven box (i.e., the Cooper-pair box) acting as a quantum two-level system, showing that the time correlations present at the detector output violate the inequality.

\section{Kochen-Specker theorem}

This theorem elucidates the conflict between quantum mechanics and noncontextual hidden-variable theories [92]. Noncontextuality means that the measured value of an observable is independent of the choice of other co-measurable (commuting) observables that are measured previously or simultaneously. Quantum mechanics is contextual, because outcomes depend on the context of measurement. This theorem is an important complement to Bell's theorem; testing it can disprove noncontextual hidden-variable theories without referring to locality. To confirm such a counterintuitive phenomenon on a macroscopic scale, it was proposed [100] to use two charge qubits which are controllably coupled by a two-level data-bus built from a phase qubit. The analysis [100] showed that by performing joint nondestructive quantum measurements of two distinct qubits, the proposed superconducting circuits could demonstrate quantum contextuality at a macroscopic level. 
For a system consisting of a superconducting qubit in an array of coupled cavities, the photon transmission exhibits a more general line shape [72], beyond the Breit-Wigner and Fano line shapes, because of the nonlinear photonic dispersion relation. At a particular matching condition between the photon wavelength and the lattice constant [72], the photonic dispersion relation can become linear and the photon transmission has the Breit-Wigner line shape, just as in an open transmission line [74]. Recently, this phenomenon was observed in a superconducting flux qubit coupled to an open transmission line [75]. When two superconducting qubits are placed in an array of coupled cavities, they can be used as tunable mirrors to form a Fabry-Perot interferometer [73]. Such a controllable on-chip interferometer is expected to have various applications in quantum optics.

Quantum nondemolition measurements. In a quantum measurement, a signal observable of a quantum system is measured by detecting the change in an observable of the detector that is coupled to the quantum system during the process of measurement. Generally, the process of measurement will disturb the state of the quantum system owing to the interplay between the system and the detector. A quantum nondemolition (QND) measurement does not perturb the subsequent evolution of the quantum system; this can be achieved by using a particular type of system-detector coupling that preserves the eigenstates of the signal observable in the quantum system. In quantum optics, a QND measurement of the photon number can be implemented using the optical Kerr effect and a dispersive atom-field coupling (see, e.g., [29]).

The first successful QND measurement on a superconducting qubit was implemented using the dispersive atom-field coupling technique $[16,76]$. Recent experiments [77, 78] show that QND measurements can also be implemented for a single superconducting qubit by using a nonlinear resonator as the detector. In [77], the detector was composed of a SQUID shunted with a capacitance, while in [78] the detector was a bifurcation amplifier, which is a r.f.-driven Josephson junction working near the dynamical bifurcation point [79]. Very recently, a fast QND measurement of a flux qubit was implemented in the weakly-projective regime by employing a hysteretic d.c. SQUID detector [80]. A quantum device can have multiple qubits, so QND measurements on quantum states of multiple qubits (e.g., entangled states) should be an appealing topic for future investigations.

Generating squeezed states. Squeezed states have been extensively studied in quantum optics, and are now being studied in condensed matter systems. Owing to their tunable nonlinearity and low losses in the microwave regime, Josephson-junction superconducting circuits are promising devices for producing squeezed states. In superconducting circuits, $L C$ oscillators have been successfully used for quantum control and readout devices in conjunction with superconducting qubits. As parametric transducers (essentially, a radio-frequency auto-oscillator), superconducting resonant tank circuits have been used to measure the quantum state of flux qubits [81]. When squeezed states are generated in these resonant tank circuits acting as quantum-state detectors, the noise of the detectors can be decreased below the standard quantum limit. A recent theoretical study [82] shows that a superconducting parametric transducer can naturally implement this approach, as it can be used both to produce squeezed states and to use them in order to minimize quantum fluctuations. An immediate application of this method would be to suppress the effective noise temperature of the amplifier connected to the parametric transducer, at least to the nominal temperature of the cooling chamber.

Topological phases. A topologically-protected quantum state degeneracy cannot be lifted by any local perturbations [83]. It is therefore natural to consider using topological phases for applications requiring a high degree of quantum coherence. With superconducting circuits as building blocks, various artificial lattices can be constructed that possess interesting topological phases. For instance, it has been proposed that a triangular Josephson junction array may have a two-fold degenerate ground state, which could be used for constructing topologically-protected qubits [84]. Recently, an experiment [85] was implemented for a prototype device that consisted of twelve physical qubits made of nanoscale Josephson junctions. Owing to properly tuned quantum fluctuations, this system was protected against magnetic flux variations well beyond linear order. This suggests that topologically protected superconducting qubits are feasible. Also, superconducting circuits were proposed [86] as a way to construct the Kitaev honeycomb model, which requires that the spin (natural or artificial) at each node of a honeycomb lattice interacts with its three nearest neighbors through three different types of interactions [87]. Depending on the bond parameters, this anisotropic spin model supports both Abelian and non-Abelian anyons, which are particles obeying unusual statistics (they are neither bosons nor fermions). Its realization would provide exciting opportunities for experimentally demonstrating anyons.

Final remarks. The superconducting circuits that we have described above contain Josephson junctions that can act as nonlinear inductors. Using suitably designed superconducting circuits, it is therefore possible to fabricate field-controlled nonlinear resonators, which can be used to demonstrate the Kerr effect (either quadratic electro-optic or quadratic magneto-optic). If such circuits were used as a Kerr medium, one could carry out a 
variety of nonlinear optics experiments, e.g., coupling microwave photons, implementing quantum gates for photon qubits, and performing QND measurement. Superconducting circuits could have many other applications. For instance, it has been suggested that a coplanar waveguide with the center conductor replaced by an array of SQUIDs could be used to simulate Hawking radiation [88]. Indeed, superconducting circuits have the advantage of enabling the study of complex controllable quantum dynamics. This could lead to quantum simulations and on-chip studies of many-body physics. Numerous new phenomena and applications will continue to be discovered using superconducting circuits, and these will play an important part in future quantum technologies.

[1] Makhlin, Y., Schön, G. \& Shnirman, A. Quantum-state engineering with Josephson-junction devices. Rev. Mod. Phys. 73, 357-400 (2001).

[2] You, J.Q. \& Nori, F. Superconducting circuits and quantum information. Phys. Today 58(11), 42-47 (2005).

[3] Clarke, J. \& Wilhelm, F.K. Superconducting quantum bits. Nature 453, 1031-1042 (2008).

A review of superconducting circuits as qubits.

[4] Schoelkopf, R.J. \& Girvin, S.M. Wiring up quantum systems. Nature 451, 664-669 (2008).

[5] Nakamura, Y., Pashkin, Yu.A. \& Tsai, J.S. Coherent control of macroscopic quantum states in a singleCooper-pair box. Nature 398, 786-788 (1999).

[6] van der Wal, C.H. et al. Quantum superposition of macroscopic persistent-current states. Science 290, 773777 (2000).

[7] Vion, D. et al. Manipulating the quantum state of an electrical circuit. Science 296, 886-889 (2002).

[8] Yu, Y., Han, S.Y., Chu, X., Chu, S.I. \& Wang, Z. Coherent temporal oscillations of macroscopic quantum states in a Josephson junction. Science 296, 889-892 (2002).

[9] Martinis, J.M., Nam, S., Aumentado, J. \& Urbina, C. Rabi oscillations in a large Josephson-junction qubit. Phys. Rev. Lett. 89, 117901 (2002).

[10] You, J.Q., Hu, X., Ashhab, S. \& Nori, F. Lowdecoherence flux qubit. Phys. Rev. B 75, 140515 (2007).

[11] Steffen, M. et al. High-coherence hybrid superconducting qubit. Phys. Rev. Lett. 105, 100502 (2010).

Report of a low-decoherence flux qubit experiment.

[12] Koch, J. et al. Charge-insensitive qubit design derived from the Cooper pair box. Phys. Rev. A 76, 042319 (2007).

[13] You, J.Q. \& Nori, F. Quantum information processing with superconducting qubits in a microwave field. Phys. Rev. B 68, 064509 (2003).

[14] Blais, A., Huang, R.-S., Wallraff, A., Girvin, S.M. \& Schoelkopf, R.J. Cavity quantum electrodynamics for superconducting electrical circuits: An architecture for quantum computation. Phys. Rev. A 69, 062320 (2004).

[15] Chiorescu, I. et al. Coherent dynamics of a flux qubit coupled to a harmonic oscillator. Nature 431, 159-162
(2004).

Report of the strong-coupling regime between a superconducting flux qubit and a resonator composed of an inductance and a capacitance.

[16] Wallraff, A. et al. Strong coupling of a single photon to a superconducting qubit using circuit quantum electrodynamics. Nature 431, 162-167 (2004).

Report of the strong-coupling regime between a superconducting charge qubit and a coplanar waveguide resonator composed of a transmission line.

[17] Fragner, A. et al. Resolving vacuum fluctuations in an electrical circuit by measuring the Lamb shift. Science 28, 1357-1360 (2008).

[18] Devoret, M.H., Girvin, S. \& Schoelkopf, R. CircuitQED: How strong can the coupling between a Josephson junction atom and a transmission line resonator be? Ann. Phys. (Leipzig) 16, 767-779 (2007).

[19] Zueco, D., Reuther, G.M., Kohler, S. \& Hänggi, P. Qubit-oscillator dynamics in the dispersive regime: Analytical theory beyond the rotating-wave approximation. Phys. Rev. A 80, 033846 (2009).

[20] Ashhab, S. \& Nori, F. Qubit-oscillator systems in the ultrastrong-coupling regime and their potential for preparing nonclassical states. Phys. Rev. A 81, 042311 (2010).

[21] Nataf P. \& Ciuti, C. Vacuum degeneracy of a circuit QED system in the ultrastrong coupling regime. Phys. Rev. Lett. 104, 023601 (2010).

[22] Niemczyk, T. et al. Circuit quantum electrodynamics in the ultrastrong-coupling regime. Nature Phys. 6, 772776 (2010).

[23] Forn-Diaz, P. et al. Observation of the Bloch-Siegert shift in a qubit-oscillator system in the ultrastrong coupling regime. Phys. Rev. Lett. 105, 237001 (2010).

[24] Wilson, C.M. et al. Coherence times of dressed states of a superconducting qubit under extreme driving. Phys. Rev. Lett. 98, 257003 (2007).

Report of dressed states of a superconducting charge qubit and an intense microwave field.

[25] Liu, Y.X., You, J.Q., Wei, L.F., Sun, C.P. \& Nori, F. Optical selection rules and phase-dependent adiabatic state control in a superconducting quantum circuit. Phys. Rev. Lett. 95, 087001 (2005).

Analysis of parity symmetry and selection rules in flux qubit circuits.

[26] Deppe, F. et al. Two-photon probe of the JaynesCummings model and controlled symmetry breaking in circuit QED. Nature Phys. 4, 686-691 (2008).

[27] de Groot, P.C. et al. Selective darkening of degenerate transitions demonstrated with two superconducting quantum bits, Nature Phys. 6, 763-766 (2010)

[28] Harris, S.E. Electromagnetically induced transparency. Phys. Today 50(7), 36-42 (1997).

[29] Scully M.O. \& Zubairy, M.S. Quantum Optics (Cambridge University Press, 1997).

[30] You, J.Q., Liu, Y.X., Sun, C.P. \& Nori, F. Persistent single-photon production by tunable on-chip micromaser with a superconducting quantum circuit. Phys. Rev. B 75, 104516 (2007).

[31] Murali, K.V.R.M., Dutton, Z., Oliver, W.D., Crankshaw, D.S. \& Orlando, T.P. Probing decoherence with electromagnetically induced transparency in superconductive quantum circuits. Phys. Rev. Lett. 93, 
087003 (2004).

[32] Dutton, Z., Murali, K.V.R.M., Oliver, W.D. \& Orlando, T.P. Electromagnetically induced transparency in superconducting quantum circuits: Effects of decoherence, tunneling, and multilevel crosstalk. Phys. Rev. B 73, 104516 (2006).

[33] Ian, H., Liu, Y.X. \& Nori, F. Tunable electromagnetically induced transparency and absorption with dressed superconducting qubits. Phys. Rev. A 81, 063823 (2010).

[34] Sillanpää, M.A. et al. Autler-Townes effect in a superconducting three-level system. Phys. Rev. Lett. 103, 193601 (2009).

[35] Abdumalikov, Jr., A.A. et al. Electromagnetically induced transparency on a single artificial atom. Phys. Rev. Lett. 104, 193601 (2010).

[36] Rodrigues, D.A., Imbers, J. \& Armour, A.D. Quantum dynamics of a resonator driven by a superconducting single-electron transistor: A solid-state analogue of the micromaser. Phys. Rev. Lett. 98, 67204 (2007).

[37] Hauss, J., Fedorov, A., Hutter, C., Shnirman, A. \& Schön, G. Single-qubit lasing and cooling at the Rabi frequency. Phys. Rev. Lett. 100, 037003 (2008).

[38] Ashhab, S., Johansson, J.R., Zagoskin, A.M. \& Nori, F. Single-artificial-atom lasing using a voltage-biased superconducting charge qubit. New J. Phys. 11, 023030 (2009).

[39] Astafiev, O. et al. Single artificial-atom lasing. Nature 449, 588-590 (2007).

[40] Grajcar, M. et al. Sisyphus cooling and amplification by a superconducting qubit. Nature Phys. 4, 612-616 (2008).

[41] Meystre, P. Atom Optics (Springer, New York, 2001).

[42] Valenzuela, S.O. et al. Microwave-induced cooling of a superconducting qubit. Science 314, 1589-1592 (2006). Report of cooling for a flux qubit using an inverse process of state population inversion.

[43] You, J.Q., Liu, Y.X. \& Nori, N. Simultaneous cooling of an artificial atom and its neighboring quantum system. Phys. Rev. Lett. 100, 047001 (2008).

[44] Huang, X.M.H., Zorman, C.A., Mehregany, M. \& Roukes, M.L. Nanodevice motion at microwave frequencies. Nature 421, 496 (2003).

[45] Martin, I., Shnirman, A., Tian, L. \& Zoller, P. Groundstate cooling of mechanical resonators. Phys. Rev. B 69, 125339 (2004).

[46] Zhang, P., Wang, Y.D. \& Sun, C.P. Cooling mechanism for a nonmechanical resonator by periodic coupling to a Cooper pair box. Phys. Rev. Lett. 95, 097204 (2005).

[47] Marquardt, F., Chen, J.P., Clerk, A.A. \& Girvin, S.M. Quantum theory of cavity-assisted sideband cooling of mechanical motion. Phys. Rev. Lett. 99, 093902 (2007).

[48] Grajcar, M., Ashhab, S., Johansson, J.R. \& Nori, F. Lower limit on the achievable temperature in resonatorbased sideband cooling. Phys. Rev. B 78, 035406 (2008).

[49] Naik, A. et al. Cooling a nanomechanical resonator with quantum back-action. Nature 443, 193-196 (2006).

[50] Rocheleau, T. et al. Preparation and detection of a mechanical resonator near the ground state of motion. $\mathrm{Na}$ ture 463, 72-75 (2010).

[51] O'Connell, A.D. et al. Quantum ground state and single-phonon control of a mechanical resonator. Nature 464, 697-703 (2010).

[52] Sillanpää, M.A., Park, J.I. \& Simmonds, R.W. Coher- ent quantum state storage and transfer between two phase qubits via a resonant cavity. Nature 449, 438442 (2007).

Report of quantum-information transfer between two superconducting qubits using single photons generated in a cavity as a quantum bus.

[53] Houck, A.A. et al. Generating single microwave photons in a circuit. Nature 449, 328-331 (2007).

[54] Hofheinz, M. et al. Generation of Fock states in a superconducting quantum circuit. Nature 454, 310-314 (2008).

[55] Hofheinz, M. et al. Synthesizing arbitrary quantum states in a superconducting resonator. Nature 459, 546549 (2009).

Report of the controllable and deterministic generation of complex superposition of states with different number of photons by using superconducting circuits.

[56] Law, C.K. \& Eberly, J.H. Arbitrary control of a quantum electromagnetic field. Phys. Rev. Lett. 76, 10551058 (1996).

[57] Liu, Y.X., Wei, L.F. \& Nori, F. Generation of nonclassical photon states using a superconducting qubit in a microcavity. Europhys. Lett. 67, 941-947 (2004).

[58] Wang, H. et al. Measurement of the decay of Fock states in a superconducting quantum circuit. Phys. Rev. Lett. 101, 240401 (2008).

[59] Wang, H. et al. Deterministic entanglement of photons in two superconducting microwave resonators. Phys. Rev. Lett. 106, 060401 (2011).

[60] Liu, Y.X., Wei, L.F., \& Nori, F. Tomographic measurements on superconducting qubit states. Phys. Rev. B 72, 014547 (2005).

[61] Steffen, M. et al. State tomography of capacitively shunted phase qubits with high fidelity. Phys. Rev. Lett. 97, 050502 (2006).

[62] Katz, N. et al. Coherent state evolution in a superconducting qubit from partial-collapse measurement. Science 312, 1498-1500 (2006).

[63] Steffen, M. et al. Measurement of the entanglement of two superconducting qubits via state tomography. Science 313, 1423-1425 (2006).

[64] Filipp, S. et al. Two-qubit state tomography using a joint dispersive readout. Phys. Rev. Lett. 102, 200402 (2009).

[65] Neeley, M. et al. Process tomography of quantum memory in a Josephson-phase qubit coupled to a two-level state. Nature Phys. 4, 523-526 (2008).

[66] Bialczak, R.C. et al. Quantum process tomography of a universal entangling gate implemented with Josephson phase qubits. Nature Phys. 6, 409-413 (2010).

[67] Johansson, J.R., Johansson, G., Wilson, C.M. \& Nori, F. Dynamical Casimir effect in a superconducting coplanar waveguide. Phys. Rev. Lett. 103, 147003 (2009).

[68] Wei, L.F., Johansson, J.R., Cen, L.X., Ashhab, S. \& Nori, F. Controllable coherent population transfers in superconducting qubits for quantum computing. Phys. Rev. Lett. 100, 113601 (2008).

[69] Rangelov, A.A. et al. Stark-shift-chirped rapidadiabatic-passage technique among three states. Phys. Rev. A 72, 053403 (2005).

[70] Johnson, B.R. et al. Quantum non-demolition detection of single microwave photons in a circuit. Nature Phys. 6, 663-667 (2010). 
[71] Shevchenko, S.N., Ashhab, S. \& Nori, F. LandauZener-Strückelberg interferometry. Phys. Rep. 492, 1-30 (2010).

[72] Zhou, L., Gong, Z.R., Liu, Y.X., Sun, C.P. \& Nori, F. Controllable scattering of a single photon inside a onedimensional resonator waveguide. Phys. Rev. Lett. 101, 100501 (2008).

[73] Zhou, L., Dong, H., Liu, Y.X., Sun, C.P. \& Nori, F. Quantum supercavity with atomic mirrors. Phys. Rev. A 78, 063827 (2008).

[74] Shen J.T. \& Fan, S. Coherent single photon transport in a one-dimensional waveguide coupled with superconducting quantum bits. Phys. Rev. Let. 95, 213001 (2005).

[75] Astafiev, O. et al. Resonance fluorescence of a single artificial atom. Science, 327, 840-843 (2010).

[76] Wallraff, A. et al. Approaching unit visibility for control of a superconducting qubit with dispersive readout. Phys. Rev. Lett. 95, 060501 (2005).

[77] Lupascu, A. et al. Quantum non-demolition measurement of a superconducting two-level system. Nature Phys. 3, 119-125 (2007).

[78] Boulant, N. et al. Quantum nondemolition readout using a Josephson bifurcation amplifier. Phys. Rev. B 76, 014525 (2007).

[79] Siddiqi, I. et al. RF-driven Josephson bifurcation amplifier for quantum measurement. Phys. Rev. Lett. 93, 207002 (2004).

[80] Picot, T., Schouten, R., Harmans, C.J.P.M. \& Mooij, J.E. Quantum nondemolition measurement of a superconducting qubit in the weakly projective regime. Phys. Rev. Lett. 105, 040506 (2010).

[81] Il'ichev, E. et al. Continuous monitoring of Rabi oscillations in a Josephson flux qubit. Phys. Rev. Lett. 91, 097906 (2003).

[82] Zagoskin, A.M., Il'ichev, E., McCutcheon, M.W., Young, J.F. \& Nori, F. Controlled generation of squeezed states of microwave radiation in a superconducting resonant circuit. Phys. Rev. Lett. 101, 253602 (2008).

[83] Nayak, C., Simon, S.H., Stern, A., Freedman, M. \& Das Sarma, S. Non-Abelian anyons and topological quantum computation. Rev. Mod. Phys. 80, 1083 (2008),

[84] Ioffe, L.B. et al. Topologically protected quantum bits using Josephson junction arrays. Nature 415, 503-506 (2002).

[85] Gladchenko, S. et al. Superconducting nanocircuits for topologically protected qubits. Nature Phys. 5, 48-53 (2009).

[86] You, J.Q., Shi, X.F., Hu, X. \& Nori, F. Quantum emulation of a spin system with topologically protected ground states using superconducting quantum circuits. Phys. Rev. B 81, 014505 (2010).

[87] Kitaev, A. Anyons in an exactly solved model and be- yond. Ann. Phys. (N.Y.) 321, 2-111 (2006).

[88] Nation, P.D., Blencowe, M.P., Rimberg, A.J. \& Buks, E. Analogue Hawking radiation in a dc-SQUID array transmission line. Phys. Rev. Lett. 103, 087004 (2009).

[89] Friedman, J.R., Patel, V., Chen, W., Tolpygo, S.K. \& Lukens, J.E. Quantum superposition of distinct macroscopic states. Nature 406, 43-46 (2000).

[90] Bertet, P. et al. Dephasing of a superconducting qubit induced by photon noise. Phys. Rev. Lett. 95, 257002 (2005).

[91] Simmonds, R.W. et al. Decoherence in Josephson phase qubits from junction resonators. Phys. Rev. Lett. 93, 077003 (2004).

[92] Genovese, M. Research on hidden variable theories: A review of recent progresses. Phys. Rep. 413, 319-396 (2005).

[93] Wei, L.F., Liu, Y.X. \& Nori, F. Testing Bell's inequality in a constantly coupled Josephson circuit by effective single-qubit operations. Phys. Rev. B 72, 104516 (2005).

[94] Kofman A.G. \& Korotkov, A.N. Analysis of Bell inequality violation in superconducting phase qubits. Phys. Rev. A 77, 104502 (2008).

[95] Ansmann, M. et al. Violation of Bell's inequality in Josephson phase qubits. Nature 461, 504-506 (2009).

[96] Neeley, M. et al. Generation of three-qubit entangled states using superconducting phase qubits. Nature $\mathbf{4 6 7}$, 570-573 (2010).

[97] DiCarlo, L. et al. Preparation and measurement of three-qubit entanglement in a superconducting circuit. Nature 467, 574-578 (2010).

[98] Leggett, A.J. \& Garg, A. Quantum mechanics versus macroscopic realism: Is the flux there when nobody looks? Phys. Rev. Lett. 54, 857-860 (1985).

[99] Palacios-Laloy, A. et al. Experimental violation of a Bell's inequality in time with weak measurement. $\mathrm{Na}$ ture Phys. 6, 442-447 (2010).

[100] Wei, L.F., Maruyama, K., Wang, X.B., You, J.Q. \& Nori, F. Testing quantum contextuality with macroscopic superconducting circuits. Phys. Rev. B 81, 174513 (2010).

Acknowledgments We thank S. Ashhab for comments on the manuscript. J.Q.Y. acknowledges partial support from the National Basic Research Program of China grant No. 2009CB929300, the National Natural Science Foundation of China grant No. 10625416, the ISTCP grant No. 2008DFA01930, and the MOE grant No. B06011. F.N. acknowledges partial support from the Laboratory of Physical Sciences, National Security Agency, Army Research Office, DARPA, AFOSR, National Science Foundation grant No. 0726909, JSPS-RFBR contract No. 09-02-92114, Grant-inAid for Scientific Research (S), MEXT Kakenhi on Quantum Cybernetics, and the JSPS through its FIRST Program. 